lighter constituents and may account for some of the discrepancies in the comparison.

From the data obtained on Trinidad Lake asphalt it seems that this method applies to this natural bitumen as well as to the artificial asphalts. Moreover, a comparison of the results obtained for Mixture No. 4 with those for the Trinidad Lake asphalt shows a sufficiently close agreement to expect of them similar qualities. Determination was therefore made of their flowing and melting points with results as follows:

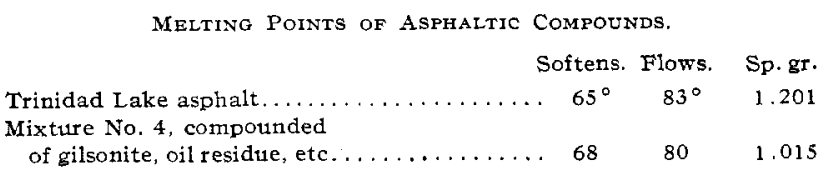

These results, it will be seen, are quite consistent with the analytical data and make it evident that if the intrinsic qualities of these substances differ, the indication for that fact must be sought in a further examination of the fractions thus obtained by precipitation.

In the sample of coal-tar, the case stands very differently in that there is no second precipitate and in its yielding a first precipitate of such different properties from that found in the true asphalts as to make its detection a simple matter.

Much additional work needs to be done in the matter of the further examination of the various fractions to differentiate still further their properties, if indeed there are differences as between the various types of asphaltic compounds.

The method of separation as it now stands is no doubt susceptible of further improvement, but in its present state its advantages over the usual dissolving-out process are very pronounced. The use of hexane has been' adopted because it is more nearly a constant compound than the heavier distillates. The amount of Precipitate No. I bears a direct relation to the specific gravity of the precipitating solution, a higher quantity being recovered with hexane than with ligroin, or the heavier distillates. While these latter are much cheaper, they vary not only from one lot to another, but frequently in the mere matter of handling, a modification in density will take place. For this reason the hexane is more satisfactory.

We wish to express our appreciation for materials furnished us gratuitously by the Standard Asphalt and Rubber Company, Barber Asphalt Company, and Standard Oil Company.
REFERENCES.

I Boussingault, J. B., Ann. de Chim, et Phys., 64, I4I ( 1837 ).

2 Le Bel, Bull. Soc. Chim., 50, 359 (I883).

3 Richardson, Clifford, "Modern Asphalt Pavement."

4 Linton, Laura A., Jour. Am. Chem. Soc., I6, 809 (1894); I 8, 275 (1 896).

5 Marcusson, A., and Eichman, R., Chem. Ztg., 32, 965 (Oct. 3, 1895).

6 Sadtler, S. P., Jour. Frank. Inst, I40, 385 (1895).

7 Endemann, Jour. Soc. Chem. Ind., 1896, 874; Municipal Engineer, 13, 6.

8 Tillson, Trans. Am. Soc. Civil Eng., 1897, 2 I4.

9 Sadtler, "Industrial Organic Chemistry."

Io Day, Jour. Frank. Inst., Sept., 1895.

I I Hodgson, E. H., Jour. Am. Chem. Soc, I898, 882.

I2 Peckham, Jour. Frank. Inst, 1896.

I3 Sabin, Jour. Am. Chem. Soc., I896, 283.

I4 Lunge, G., and Krepelka, V., Jour. Soc. Chem. Ind., I $904,436$.

CNIVERSITY OF ILLINOIS URBANA, ILLINOIS.

[CONTRIBUtion No, 9 fRom 'THe Research LABORATORy OF APplied Chemistry, Mass. Inst, of Technology.]

\section{PAINT AND VARNISH COATINGS AS ACCELER- ATORS IN THE CORROSION OF METALS.}

By WilliaM H. WALker AND WARREN $K$. LEWIS.

Received July 1, 1909.

The packing of strongly acid fruits in tin cans has always been attended with more or less difficulty. A new method which has recently been developed for the protection of the can from the action of the fruit juices, is to lacquer the inside of the can with a high-grade copal-linseed oil varnish. The results are excellent so far as the preservation of the fruit is concerned, but an entirely unexpected difficulty has been met; namely, that the cans sometimes corrode through within a short time (six weeks or two months) after packing. An investigation of the nature of the varnish coating and the cause of its accelerating action on the corrosion of iron and tin has been carried out in this laboratory and has led to some very interesting results, which are the subject-matter of this paper.

The cans, placed at our disposal, were lacquered while still in the sheet, on one side, by a process analogous to that used in lithography. The lacquer was baked at $\mathrm{I}_{40}-\mathrm{r}_{5} \mathrm{O}^{\circ} \mathrm{C}$. and the cans made up in the ordinary way from these sheets, the lacquered surface being inside. The interior of new cans was of a light, golden yellow color, and the coating to the eye was perfect in appearance. Cans which had been packed with strawberries for six to eight weeks were, however, badly corroded 
on the interior. The corrosion was concentrated, mainly at the soldered joints, at those points where the die had crimped the ends, and along straight parallel lines down the sides of the cans. The action was extremely serious, the attacked areas being deeply eaten away, in some points the metal being corroded through, with a resultant leak.

While it is true that certain very acid fruits attack an ordinary can to a certain extent, it is no less true that under ordinary conditions the total corrosion, despite the fact that the available corroding surface is greater, is decidedly less than is the case in a lacquered can. It is impossible, then, that this corrosion of the lacquered can is due entirely to the acid of the fruits. The only new factor introduced is the lacquer itself and it must be that the lacquer in some way causes or accelerates corrosion. According to the electrolytic theory of the corrosion of metals, the rate of reaction can be increased only in three ways, either ( $\mathrm{I}$ ) by decreasing the concentration of the metal ion in the electrolyte in contact with the surface, (2) by increasing the concentration of the hydrogen ion about the cathodic area, and in these two ways increasing the driving force of the reaction, or (3) by lessening the polarization of the deposited hydrogen by the use of some effective depolarizing agent. The only way in which the lacquer could alter the ionic concentration of the two metals, tin and iron, involved, would be by their absorption and consequent elimination from the solution. It is in the first place extremely improbable that the lacquer should do this to any appreciable extent and then again we know that since the rate of corrosion of both these metals in pure water is so extremely small, that this absorption, even though complete, would have but a slight effect on the rate of reaction. That the lacquer should increase the acidity of the electrolyte materially above that due to the acids of the fruit juices is equally improbable, and, consequently, we are driven to the conclusion that it must be as a depolarizer that the lacquer exerts its influence. This is made the more likely by the fact that depolarization is by far the most effective means of accelerating the corrosion of metals in general. It must be that the lacquer renders the corrosion of the cans possible by the absorption and removal of the depolarizing hydrogen from the cathodic surface.

All protective films made from so-called drying oils owe their properties to the unsaturated nature of those oils and their consequent ability to absorb oxygen, transforming themselves thereby into substances such as linoxylin and related compounds. As is well known, this absorption is a slow one and the unsaturated state disappears completely only after the lapse of a great length of time, unless accelerated by the use of catalyzers (artificial driers), or by the increase of the reaction velocity through the use of higher temperature. Thus it is not only possible but frequent that such films even in use are still partly unsaturated. It is equally well known that unsaturated carbon compounds in general possess not only a capacity for absorbing oxygen, but hydrogen as well, and the unsaturated state can be relieved equally well by reducing agents as by the use of oxidizing materials. We know, however, that such compounds are capable of absorbing hydrogen only in the nascent condition; it is then not surprising if a linoxylin film, which is as yet not completely saturated, will absorb the polarizing hydrogen deposited when a metal such as iron or tin is brought in contact with an electrolyte.

To investigate the accuracy of these deductions the following experiments were performed: A Utube containing $\mathrm{KCl}$ solution with an agar plug at the bottom, from which air had been carefully expelled, had inserted into one arm a lacquered electrode and into the other, one of bare iron, the two being connected externally. While blanks showed no test for iron whatever, when one electrode was lacquered with an oil and varnish film, a strong test for iron was obtained in the other arm of the U-tube. The corrosion was greatest with a linoxylin film; copal-varnish, cellulose nitrate, shellac, and asphalt followed in order, while lacquer baked sufficiently long at $250 \mathrm{C}$., as well as paraffine, showed no corrosion whatever.

In order to make sure that the corrosion was not due to small amounts of air which it might have been possible to remove from the agar plug in the bottom of the $U$ tube, or which might condense on the walls of the glass which could not be heated up after the setting of the agar, the experiment was repeated in an apparatus which precluded the possibility of such an error. In a Mason jar, thoroughly steamed out for forty-eight hours before use, were placed two concentric, porous, porcelain cups and the whole three-fourths filled with water, which was boiled down to about one-third the volume of the jar. The electrodes were inserted into the boiling solution and the cap, through which lead a tube delivering hydrogen, was screwed on while 
steam was still escaping at full force from the jar. Numerous experiments have shown that under these conditions, with bare electrodes, sufficient iron to be detected with ferricyanide is never obtained. If, however, a lacquered electrode was inserted into the outside compartment and a bare iron electrode in metallic contact with it into the inner cup, the inner cup invariably showed a strong test for iron after from eighteen to twenty-four hours. No iron could be detected in either the jar or the larger cup.

If such films accelerate corrosion, it must be made possible only by the flow of an electric current, and this current must be carried either by the conductivity of the film as such, or by the solution penetrating through its pores to the metallic surface beneath. The conductivities of such films were tested by measuring the resistance of a circuit consisting of a metallic conductor leading to two electrodes immersed in a $\mathrm{U}$ tube, the resistance being measured before and after coating with the film in question. The electrodes employed were short iron wires and the electrolyte a strong solution of calcium chloride. The vessel was a small $U$ tube. The resistance of the circuit with bare electrodes was approximately $30 \mathrm{ohms}$. Lacquered electrodes increased the resistance to some $270 \mathrm{ohms}$ but in the course of a couple of hours this value had fallen to about 80 ohms. In the same time, shellac presented a resistance of 70 ohms, cellulose nitrate of 80 , linoxylin of 65 , asphalt of 43 , while paraffine electrodes offered a resistance so great that it could not be measured with the apparatus at hand. In other words, paraffine was impervious to the solution employed. By the addition of paraffine to the lacquer, its resistance could be increased to from $1_{500-3000}$ ohms, but the lacquer had become so brittle thereby that the practical use of such a film would be out of the question.

To show that the corrosion of the iron occasioned by the lacquer film is accompanied by the flow of an electric current through the external circuit, the following experiment was tried: A U-tube, as previously described, with a $\mathrm{KCl}$ solution and an agar plug, carefully boiled out, the whole under an atmosphere of hydrogen, contained two iron electrodes, the one bare and the other covered with a linoxylin film. An electrometer was inserted into the external circuit and the current flow measured as shown in the table below. It was found undesirable to use a silver voltameter for the excessively small currents employed and resort was had to a modification of Ostwald's Bromide Voltameter, described elsewhere in the Journal of this Society. This voltameter was used for all measurements made, after the first, which was done with the silver nitrate instrument.

Deposit obtained from current due to depolarization by two different linoxylin films.

$\begin{array}{cccc} & \begin{array}{c}\text { Time in } \\ \text { hrs. }\end{array} & \begin{array}{c}\text { Deposit of } \\ \text { mgs. bromine. }\end{array} & \begin{array}{c}\text { Deposit mgs. } \\ \text { per hrs. }\end{array} \\ \text { Film No. 1 } & 48 & 0.6 & 0.012 \\ \text { Film No. } 2 & 76 & 1.0 & 0.013 \\ & 41 & 1.8 & 0.044 \\ & 89 & 4.1 & 0.046 \\ & 185 & 8.7 & 0.047\end{array}$

These figures prove beyond doubt that the corrosion of the iron on the unlacquered side is due to the flow of an electric current through the external circuit from the lacquered surface.

These experimental facts seem to allow of but one explanation, namely, that the films in question are porous in their nature, allowing the electrolyte to penetrate them to the surface of the metal beneath, that some of these films, due to their unsaturated state, are capable of absorbing nascent hydrogen and in this way acting as depolarizers, and that aside from this, all the porous films allow the penetration through them to the surface beneath of any depolarizer that may exist in the solution, thus rendering the coated surface cathodic and concentrating the solvent action at the exposed part of the metal.

The reason for the failure of the fruit cans mentioned at the beginning of this article is now easily understood. While the lacquer film applied to the uncut sheet was probably a perfect one, still in the process of making the can, this film was ruptured at many points. Thus the die stamping out the head of the can broke the film at the place where the tin was bent; the mandrel, on which the body of the can was formed, scratched the sides in long parallel lines upon the removal of the can; and the burning of the joints destroyed the lacquer in their immediate neighborhood. When the fruit was introduced into the can, the depolarizing action of the lacquer itself, coupled with that of the small amount of air left in the can in packing, threw the protected areas into the cathodic state, concentrating the solution of the metal at the exposed points, dissolving in this way both tin and iron, and maintaining this corrosion until both the air was consumed and the unsaturated state of the lacquer was completely relieved. Before this point was reached, however, the corrosion 
had gone far enough to seriously damage the fruit and even in many cases to puncture the can. This action is entirely independent of any possible imperfections in the tin plate. The remedy would be to find a lacquer impervious to the solution, or if that prove impracticable to at least furnish one which will not act as a hydrogen depolarizer. A non-porous lacquer it has as yet proven impossible to find; a saturated one can be made by sufficiently baking any ordinary varnish, but there still remains work to be done to develop this into a satisfactory solution of the problem.

It is self-evident, however, that the importance of these phenomena is by no means limited to the problem of lacquering fruit cans. The majority of protective coatings for iron contain linseed oil or some one of the various substances found by our experiments to be either unsaturated, or porous, or both; and so soon as a piece of metal painted with these substances comes in contact with water after the abrasion of the paint film at any point, all the conditions for corrosion as above outlined are fulfilled, and we may be sure that corrosion at the exposed point will be accelerated by the presence of such films in its neighborhood. It is true that the porosity of these films is reduced to a minimum by the use of the best obtainable loading materials, such for instance as certain pigments of ordinary linseed paints, the bituminous bodies of asphalt or coal tar paints, etc., and that these paints offer in consequence a much greater electrical resistance to the flow of the current than otherwise. One must not forget, however, that the insertion of such a resistance to the current flow can only reduce the rate of the reaction, and in no way influence its tendency or driving force. The exposure of such films to the air for a long period of time finally entirely saturates them, but this again does not affect their porosity and consequently does not preclude the possibility of acceleration of corrosion at the exposed point due to the depolarizing action of air through the film. These facts make clear the reason for the rapid deterioration and pitting of the iron or steel surface at points laid bare by the breaking down of many paint films and show why it is so exceedingly important that a metal surface should be clean and bright before the application of a paint.

If a paint or lacquer film be intact, despite the fact of its porosity, corrosion does not seem to take place at once beneath its surface. Thus an iron can, carefully painted with a high-grade varnish and filled with cold 5 per cent. sulphuric acid, showed a test for iron with ferricyanide first after twenty-four hours. If heated on the water bath, however, a test could be obtained in slightly over one hour, but even then the action was not severe. It may perhaps be that the electrolyte does not find sufficient continuous surface beneath the film to allow of a ready separation into cathodic and anodic areas. At any rate, corrosion does not readily take place if the film be intact, but the surface below the film does easily become cathodic if an exposed area in the neighborhood can act as anode. This cathodic liberation of hydrogen loosens the film from the metal in some way not yet clearly understood and likewise softens it. The resistance of a film diminishes quite rapidly in this way and it soon becomes weak and rotten and easily removable from the iron. It is easy to see how, especially with rough usage, exposed points in a painted steel surface rapidly grow in size.

A few of the common commercial paints and paint-making materials were examined, using the following apparatus: A glass U-tube of $I^{\prime}$ tubing with $6^{\prime}$ arms and $8^{\prime}$ in length over all, contains $200 \mathrm{cc}$. of normal $\mathrm{KCl}$. The electrodes are of commercial soft iron wire, $0.044^{\prime}$ diameter carefully cleaned with emery. The bare electrode is $25 \mathrm{~cm}$. long and the lacquered one $100 \mathrm{~cm}$. The electrode is coated by dipping in the paint to be examined, the excess removed by rapid twirling and then dried. The water about the cathode is kept saturated with oxygen by bubbling air through it. The depolarization current is measured by the use of the bromide voltameter already mentioned. The bromide deposited is proportional to the time, up to a point when the film gives way. This point of disintegration is different for different films and two quantities can in general be measured, (I) the initial rate of depolarization

\footnotetext{
Paint 6lm. Cortosion in mgs. Bromine per hour.

"Durable metal coating"........... 0.70

"Copal linseed oil lacquer ".......... 0.76

"Cosmos" (coal tar) ................. 0.34

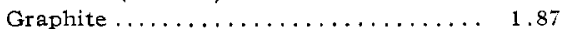

Carbon black................. 2.4

Lampblack ................... 2.3

Zinc chromate ................. $0.11^{1}$

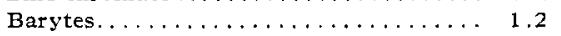

Zinc oxide ....................... $0.078^{2}$

Graphite (baked) ............... 0.078

White lead..................... $0.10^{3}$

Linoxylin .................. 0.31

Paraffine....................... 0.00

1 Broke down in 36 hours, but not badly.

2 Still perfect after 71 hours.

3 Broke down in 30 hours.
} 
and (2) the time of rupture. This second factor is somewhat difficult to obtain because our voltameter measures not current but total amount of electricity passed, and it is frequently, if not usually, impossible to tell at exactly what point the increase in current began. In the above table is the average of the data obtained.

It is evident that the electricity measured by the bromine deposited is the resultant of a number of factors acting at the same time. A comprehensive study of the relationship between such data and the value of various paints as a protective coating for iron is now in progress.

\section{THE CARTER PROCESS OF WHITE LEAD MANUFACTURE.}

By J. S. Staudt.

Received July 17, 1909.

This company has two plants in the United States: one in Chicago, Ill., and the other in Omaha, Neb. The former has a capacity of about 20,000 tons annually and the latter of about ro,000 tons annually.

All descriptions and illustrations as herein given refer to the Omaha plant.

The lead bars or pigs, weighing about $95 \mathrm{tbs}$. each, are drawn up a chain elevator at the rate of 500 pigs per day, to be melted in a cast iron trough about 8 feet long, I 8 inches wide and I 2 inches deep, placed upon the second floor of what is called the "blow room." The lead is melted by means of a coal fire placed inmmediately below the trough.

On the side of the trough opposite the elevator is an opening through which pass two concentric tubes. The inner tube, nozzle shaped, serves for the passage of molten lead, and the outer for the passage of superheated steam. The tubes lead or open into a large iron compartment, about 33 feet long and so feet high, with a maximum depth of about Io feet, tapering toward the base. Superheated steam is here produced by the same source of heat used in melting the lead, and is thus made as needed. It is the action of this superheated steam, blown against the molten lead, blowing it against the walls of the compartment, that reduces it into a finely divided state, granular and blue in appearance, called "blue lead."

The blown lead is not supposed to be oxidized before being put into the corroding cylinders. It will, however, undergo a slight oxidation when subjected to the moist air and the gases of the plant. When heaped up it will rise in temperature and become partly oxidized. Trap doors at the bottom of the iron compartment serve as means for the removal of the blown lead. The blown lead is removed by means of iron trucks, mounted on cast iron wheels, each truck holding about 4,000 tbs. Each truck is hauled upon an elevator and raised to the third floor. The contents are then dumped through an opening leading into a "corroding cylinder," on the second floor beneath. All the "corroding cylinders" are filled in this way, each "blue cylinder" receiving about 4,000 tbs. of blown lead. In the "corroding room" there are 69 "blue cylinders," as they are called, and 58 "white cylinders." The cylinders are made of wooden

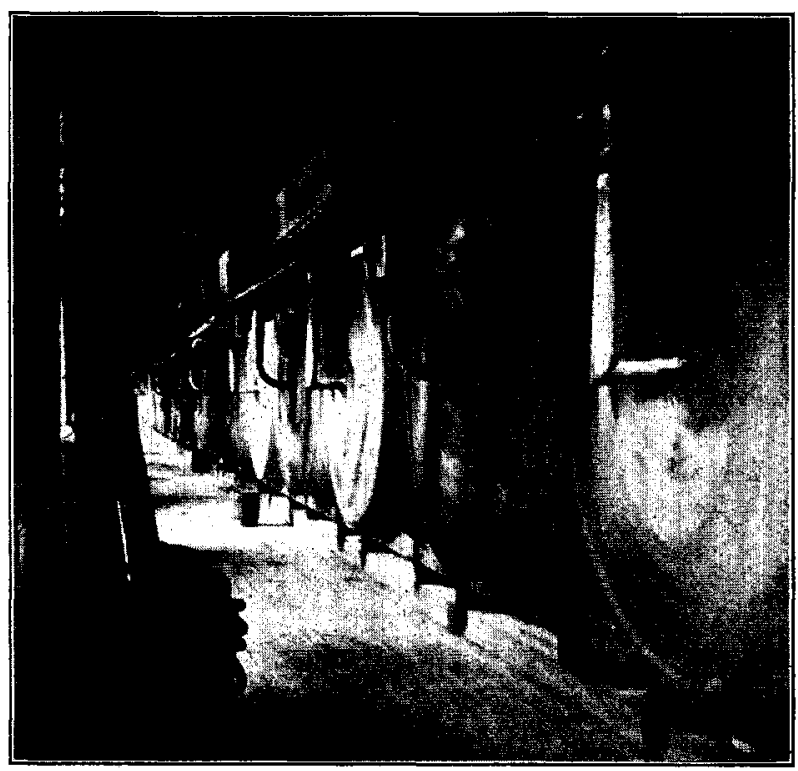

Fig. 1,-Corroding cylinders.

planks 2 inches thick, are Io feet long and 8 feet in diameter. Lead is kept in the "blue cylinders" for Io days, $16 \mathrm{lbs}$. of acetic acid being added for three days. For the remaining seven days it is sprinkled with cold water by means of a hose, inserted through an opening in the end of the cylinder. This is done twice a day, once in the morning and once in the evening. The carbon dioxide enters the cylinder through a 2 -inch pipe inserted through an opening in the axis of one end of each cylinder. The cylinders revolve very slowly, making one revolution in nine minutes. The function of the cylinders is twofold: first, it serves as a grinder in so far as the particles rub against each other while the cylinder revolves. 